\title{
¿DÓNDE LEES TÚ?: CARTOGRAFÍA DE LECTURA DE LOS CHILENOS Y CHILENAS
}

Begoña Alberdi Soto 


\section{BEGOÑA ALBERDI SOTO}

Coordinadora del Observatorio del Libro y la Lectura. Licenciada en Letras Mención Lingüística y Literatura Hispánicas, y Magíster en Literatura de la Pontificia Universidad Católica de Chile. Se ha desempeñado en el área de docencia e investigación en la Facultad de Artes y la Facultad de Letras de la misma Universidad. 


\section{¿DÓNDE LEES TÚ? \\ CARTOGRAFÍA DE LECTURA DE LOS CHILENOS Y CHILENAS}

\section{INTRODUCCIÓN}

«El "dónde" de la lectura es más importante de lo que podría creerse, pues la situación del lector en su escenario puede darnos indicios sobre la naturaleza de su experiencia» (Robert Darnton, "Historia de la lectura", en Formas de hacer Historia, Peter Burke, pág. 189)

Como parte de su misión de promover acciones de estudio y socialización de la realidad, hábitos y prácticas del libro y la lectura en Chile, el Observatorio del Libro y la Lectura ha llevado a cabo, por dos años consecutivos, el estudio de hábitos y prácticas lectoras “¿Dónde lees tú?", en el espacio de la Feria Internacional del Libro de Santiago.

A través de un cuestionario realizado a los(as) asistentes de la Feria, versión 2012 y 2013, el Observatorio se ha propuesto cartografiar los lugares habituales de lectura de los chilenos(as), con el objetivo de configurar las prácticas lectoras particulares en sujetos que leen en contextos y formatos distintos, así como analizar las prácticas rutinarias que favorecen y limitan la lectura y los espacios sociales en donde se lee.

En relación a esto último, el presente documento pretende difundir los principales resultados de la primera y segunda versión de esta encuesta que, cabe aclarar, no pueden extraerse a la población chilena total, pero sí contribuir de forma importante a conocer las especificidades en las prácticas de un grupo particularmente cercano a la lectura, como son los(as) asistentes voluntarios(as) a una Feria del Libro.

El objetivo será entregar una mirada respecto a aquellos resultados que dan cuenta de posibles transformaciones en las prácticas, lugares y gustos de lectura, considerando variables sociales altamente relevantes como género, edad, ocupación y comuna de residencia de los sujetos encuestados. Así, y dado que las prácticas lectoras están influidas por estos factores estructurales y culturales, será su dinámica concreta la que aporte las condiciones necesarias, en términos de tiempo, espacio, valoración y concepciones atribuidas a la lectura.

En este sentido, será importante observar cómo operan los espacios públicos y privados en las prácticas lectoras, así como los cambios y hallazgos que puedan dar lugar a la implementación de políticas públicas dirigidas a fomentar la lectura. Por último, este análisis busca también contribuir a la generación de preguntas e hipótesis de investigaciones a futuro, que contribuyan al conocimiento y promoción de las prácticas lectoras.

“¿Dónde lees tú?" pretende dar cuenta cómo ciertos usos y prácticas lectoras revelan, en realidad, dinámicas particulares de apropiación que dan cuenta de las desigualdades de género, edad y clase en la posesión y uso del tiempo, en la disponibilidad para realizar actividades de ocio o artísticas y en la movilidad de los sujetos en la ciudad, de acuerdo a sus ocupaciones y comunas de residencia, entre otros aspectos. 


\section{ANÁLISIS DE LOS PRINCIPALES RESULTADOS}

\subsection{La lectura y la intimidad de lo privado}

"Raras veces leo en playas o jardines. No se puede leer con dos luces al mismo tiempo, la luz del día y la del libro. Hay que leer con luz eléctrica, la habitación en sombras, solo la página iluminada".

(Marguerite Duras, en Antonio Manguel, Historia de la lectura, pág. 182)

Uno de los principales resultados que se desprende de la encuesta “¿Dónde lees tú?" es que el espacio privado sigue siendo uno de los lugares más relevantes para la lectura, práctica que se sostiene — sobre todo- en la intimidad e individualidad. En todas las edades y géneros, mujeres, hombres, jóvenes y adultos manifiestan que su lugar preferido de lectura es el dormitorio (83\% en 2012 y 86\% en 2013), preferencia que — podemos inferir- no apunta a una silla o escritorio, sino particularmente a la lectura sobre la cama.

Así, y a diferencia de aquella lectura en voz alta, altamente vinculada a procesos de socialización, la encuesta "¿Dónde lees tú?" confirma una marcada preferencia por la lectura silenciosa y visual que se realiza sobre la horizontalidad de la cama, marcando un acto de distancia y aislamiento de un individuo en relación con el resto: según Manguel, "(...) leer en la cama proporciona algo más que entretenimiento: una peculiar sensación de intimidad. Leer en la cama es un acto egocéntrico, inmóvil, libre de las ordinarias convenciones sociales, invisible para el mundo y que, por producirse entre las sábanas, en el reino de la lascivia y de la pereza pecaminosa, participa de la emoción de las cosas prohibidas".

En consonancia a los resultados de esta encuesta, la cita de Manguel nos revela que el componente de intimidad e individualidad sigue siendo central para comprender la práctica actual de la lectura en Chile. A esto se suma que, al interior del hogar, subyace una determinada distribución de los espacios según los roles y atribuciones culturalmente asumidos por los sujetos, de acuerdo a su género, edad y posición dentro de la estructura familiar.

Así, se visualizan algunas diferencias que distinguen espacios y lugares tomados por hombres y mujeres al momento de hacer uso de lo escrito: mientras un alto porcentaje de hombres lo realiza en el baño, esto es, en una situación completamente individual, por su parte, las mujeres suelen leer en la sala de estar, escritorio o jardín, es decir, en espacios con mayor conexión a la dinámica familiar. Esta información sugiere que el baño es un espacio en que los hombres no son "molestados" por la cotidianidad del hogar y de la vida doméstica, esto es, un espacio aislado y solitario, mientras que las mujeres - mediadoras entre lo público y lo privado- ocupan las habitaciones que se conectan al resto del hogar.

Asimismo, también es relevante que las mujeres leen más fuera de casa, señalando como lugares relevantes el metro, la playa, lago o montaña y las salas de espera. Resulta interesante visualizar esta diferencia, ya que las mujeres encontrarían fuera de casa un espacio propio para la lectura, dado que la sobrecarga de quehaceres domésticos y de cuidado les impediría vivir este tiempo de forma individual. Por su parte, y en relación 
a lo mencionado anteriormente, los hombres se encerrarían a leer en el baño para buscar algo de intimidad, al aislarse del contexto familiar.

Según la variable "edad", la encuesta "¿Dónde lees tú?" también encontró diferencias relevantes, acorde a la etapa de ciclo vital y ocupacional. Así, mientras el tramo de jóvenes de 18 a 29 años lee más en el Metro, micro, bibliotecas y Universidad —dado que la mayoría son estudiantes que se movilizan dentro de la ciudad y utilizan instituciones educativas y de formación-, por su parte, el tramo de adultos de 30 a 39 años lo hace más en el baño y lugar de trabajo. En este grupo etario, en el que la mayoría personas trabaja, probablemente se destina más tiempo a una lectura funcional o utilitaria asociada al trabajo, o bien a una lectura por placer en el hogar.

Por su parte, el tramo de 40 a 55 años señala leer más en lugares de ocio y vacaciones como playa, lago o montaña, salas de espera y salas de estar. De lo anterior se deriva, claramente, la existencia de una concepción de lectura por placer, asociada a lugares de ocio y al entretenimiento, aspecto mucho más marcado que en otras edades. Asimismo, adultos y adultos mayores de 56 años y más, señalan leer en mayor proporción en salas de espera y salas de estar, es decir, en instituciones donde las personas requieren entretenimiento temporal mientras esperan por un trámite o situación puntual.

Por último, en términos de clase y considerando, además, la alta segregación y desigualdad urbana de Santiago, se pueden inferir algunas conclusiones según la variable "comuna de residencia" de los sujetos encuestados. A modo de ejemplo, se observa que las personas provenientes de Las Condes, Providencia, Ñuñoa y La Florida, manifiestan practicar más la lectura en el lago, playa y montaña, es decir, en lugares de vacaciones o dedicados al ocio durante los fines de semana.

Cabe destacar que esto no significa que el grupo en cuestión no sea lector en otras circunstancias, sino que, más bien, resalta una concepción de la lectura asociada al placer como una actividad propia del ocio, divertimento o relajo, fuera del espacio mundanal de la ciudad, incluso en el imaginario. Lo interesante de esta reflexión es que este grupo de personas corresponde a lectores habituales y con condiciones de acceso a diarios, revistas y libros, pero simbólicamente no asocia la lectura al deber - a pesar de que efectivamente sí la practica en ese contexto- sino a la libertad de aquella recreación que se emprende por placer; momento en que el conocimiento y la comprensión de los contenidos del libro cobra sentido y significado de acuerdo a otros valores, necesidades y motivaciones, que lo transforman para dar lugar a nuevas actitudes, prácticas y usos.

\subsection{Leer en espacios públicos: Ciudad y lectura en movimiento}

$\mathrm{Al}$ abrir los resultados de la encuesta "¿Dónde lees tú?" según comuna del encuestado, se constata que mientras Puente Alto, La Florida y Maipú leen usualmente en el metro, La Florida y Ñuñoa lo hacen en el baño, y Maipú, en la micro.

Al respecto, cabe señalar que Puente Alto, Maipú y La Florida son las comunas con mayor población en la Región Metropolitana y poseen una heterogeneidad social bastante amplia, predominantemente grupos de ingresos medios (C3, llamados "emergentes") 
y grupos de ingresos bajos, especialmente Puente Alto. Estas comunas han sido beneficiadas recientemente con la instalación del Metro, lo cual ha contribuido a mejorar su calidad de vida y su integración física y simbólica a la ciudad.

En este contexto, los medios de transporte tienen un rol importante en las prácticas lectoras, siendo la micro, y especialmente el metro, lugares cada vez más relevantes para el desarrollo de esta actividad. En efecto, según la encuesta “¿Dónde lees tú?”, existe más lectura en vagones de tren y Metro, que en cafés, librerías y bibliotecas, espacios comúnmente asociados — y diseñados- para leer.

Esto no es de sorprender si se considera la progresiva congestión del tránsito y los altos tiempos de viaje que experimentan los(as) ciudadanos(as) pues — como ya se ha constatado- la actividad de leer se relaciona directamente con los tiempos y espacios que ellos y ellas encuentran en la vida cotidiana. Así, quienes generalmente no le conceden ni tiempo ni espacio a la lectura en sus casas ${ }^{1}$, sí lo hacen en el espacio público, lugar que se vuelve fundamental al momento de pensar en el fomento a la lectura.

En ese marco, cabría preguntarse cuál es el tipo de lectura que se produce en este tipo de espacios públicos y qué tipo de competencias desarrollan sus lectores y lectoras, al alero de innumerables interrupciones y, en más de algunas ocasiones, inhóspitas condiciones. Porque no solo ciertos libros exigen un contraste entre contenido y entorno: algunos parecen exigir posiciones particulares para leer $\mathrm{y}$, asimismo, existen ciertos lugares que están en consonancia con determinadas posturas lectoras. Como bien grafica Antonio Manguel: "Hay libros que leo en mi sillón y hay otros que leo sentado frente a mi escritorio; hay libros que leo en el metro, en tranvías o autobuses. Considero que los libros leídos en trenes tienen algo en común con los libros que se leen en sillones, quizá porque en ambos casos puedo aislarme sin dificultad de lo que me rodea" (pág. 182).

\subsection{Las bibliotecas: El desafío de su trasformación}

La encuesta “¿Dónde lees tú?” revela que espacios como los cafés y bibliotecas públicas tienen una menor importancia en términos de lectura. Como se mencionó anteriormente, se lee más en el Metro (48\% en 2012, 53\% en 2013) que en las bibliotecas (37\% en $2012,26 \%$ en 2013) 2.

Asimismo, la Segunda Encuesta de Consumo Cultural (2009) del Consejo Nacional de la Cultura y las Artes, señala que el 39,8\% de los(as) lectores(as) que leyeron al menos un libro durante los últimos 12 meses, asistió a bibliotecas durante el mismo período, mientras que un $57,4 \%$ de los(as) lectores(as) no acudió a este tipo de establecimiento

1. En efecto, según el estudio del CERLALC (2012), "Comportamiento lector y hábitos de lectura", Chile registra la más baja tasa de lectura en el tiempo libre, en contraposición a países como Argentina y España que registran un 66\% y 58\%, respectivamente (págs. 10-11).

2. Estos datos son consistentes con el estudio del CERLALC (2012), "Comportamiento lector y hábitos de lectura", donde se señala que un $26 \%$ de los chilenos y chilenas utiliza la biblioteca para leer. Si bien baja, esta cifra es significativamente más alta a la de países como España y Perú, donde solo un 5\% y $6 \%$, respectivamente, señala la biblioteca como lugar de lectura. 
durante el último año. Existe un segmento de lectores(as) que expresa no haber asistido nunca en su vida a una biblioteca y un 2,9\% de personas que han leído al menos un libro en los últimos 12 meses.

En la misma línea, el estudio de Comportamiento Lector desarrollado por Microdatos (2011) indica que la gran mayoría de los(as) encuestados(as) afirma que durante el último año nunca ha asistido a una biblioteca de tipo público (85\%), de colegio (74\%), universitaria (87\%) y especializada (96\%). Las bibliotecas de colegio y universitarias son las únicas que presentan frecuencia de asistencia diaria, aunque muy baja. Entre las principales razones por las que las personas no asisten o no frecuentan una biblioteca se encuentra la falta de tiempo (72\%), seguido de la distancia del lugar en donde se vive o trabaja (11\%) y, en menor medida, un $8 \%$ que prefiere conseguir libros por otro medio.

De acuerdo a la Segunda Encuesta de Consumo Cultural (2009), se señala que quienes van a bibliotecas son más hombres (42,5\%) que mujeres (37,5\%); los lectores jóvenes de 15 a 29 años acudieron en un 57,6\%, mientras que los lectores de 60 años y más lo hicieron en un $14,4 \%$. Por nivel socioeconómico se aprecian cifras similares de asistencia a bibliotecas, lo que significa que incluso para un público más sensibilizado con la práctica lectora, las bibliotecas han perdido importancia como espacio público de lectura.

Lo anterior es un dato no menor en la medida que algo ocurre con la oferta de servicios de las bibliotecas públicas; o bien no está cubriendo los intereses, o bien no se está ajustando a las condiciones que requieren los lectores. En este sentido, existiría cierto agotamiento de este tipo de biblioteca, dado que su figura se asocia a un tipo de lectura enciclopédica o escolarizante, cuya circulación se ha modificado a causa de las herramientas digitales y virtuales y otras fuentes de información en Internet.

Las bibliotecas públicas, municipales y de universidades constituyen espacios principalmente para la lectura obligatoria. Aun cuando existen usos excepcionales, estos espacios se configuran, en general, como extensiones de las universidades o de las oficinas, en tanto prestan condiciones para el desarrollo de trabajo en solitario y en silencio mayormente.

Tomando en cuenta estos resultados de la encuesta “¿Dónde lees tú?", y haciendo eco a las reflexiones de Roger Chartier, todo indica que la biblioteca, tal y como la entendemos hoy en día, debe rearticularse como el lugar en el que se pueda mantener el conocimiento y la comprensión de la cultura escrita en las formas que han sido y son todavía mayoritariamente las suyas hoy en día. Así, la representación electrónica de todos los textos cuya existencia no comienza con la informática, no debe significar de ninguna manera la relegación, el olvido o la destrucción de los objetos que los han portado. Más que nunca, tal vez, una de las tareas esenciales de las grandes y pequeñas bibliotecas sea recolectar, proteger y censar los objetos escritos del pasado y así hacer accesible el orden de los libros que todavía es el nuestro 3 .

3. Chartier, R., "Del códice a la pantalla, trayectorias de lo escrito", en Revista Quimera, $N^{\circ} 150$, septiembre de 1996, págs. 49. 
En torno a ello, también resulta interesante que la encuesta “¿Dónde lees tú?" haya recogido una gran cantidad de comentarios que fueron señalados libremente por los(as) encuestados(as), quienes mencionaban su libro o género favorito. Esto manifiesta, en parte, que las personas carecen de un lugar para intercambiar gustos, referencias y opiniones sobre libros, es decir, una falta de espacios de intercambio y expresión entre lectores, lo que bien podría subsanarse en las bibliotecas.

Lo anterior nos permite entonces hablar de una necesidad creciente de una comunidad de lectores, productores inventivos de sentidos singulares, que convierten la lectura en una infinidad de posibilidades o experiencias irreductibles unas de otras. De este modo, y en el marco de la biblioteca, la solución radicaría en hacer la historia de las prácticas de lectura para cada época y cada medio, identificando las modalidades compartidas del acto de leer que sitúan los gestos individuales y los procesos a través de los cuales un texto produce una significación específica, a un(a) lector(a) o a una comunidad de lectores(as).

\section{3. ¿ASISTIMOS HOY A UNA NUEVA CARTOGRAFÍA DE LECTURA?}

Antes de reflexionar acerca de si estamos o no en presencia de una nueva cartografía de lectura, es necesario destacar que una de las principales conclusiones del estudio es que el libro, en tanto bien cultural y formato, tiene plena vigencia. Contra los discursos que intentan conjurar la desaparición del libro, de lo escrito y de la lectura, en un contexto cada vez más tecnologizado, la encuesta “¿Dónde lees tú?" revela que, en realidad, el libro goza de gran vitalidad. Así, la mayor parte de los lectores y lectoras (95\% en 2012 y $92 \%$ en 2013), declara que prefiere leer libros por sobre revistas (45\% y 33\%), diarios (45\% y 42\%) y cómics (26\% y 14\%) ${ }^{4}$. Además, un $83 \%$ declara leer desde sus computadores o utilizar frecuentemente libros electrónicos.

En ese sentido, cabe destacar que, al mencionar su preferencia por el libro, los(as) encuestados(as) apuntan, indistintamente, tanto a la descripción morfológica del objeto que lo difunde - el libro impreso, la construcción de la página, las divisiones del texto, la tipografía, etc. - como al discurso abstracto del mismo, separado de una materialidad particular y vinculado a distintas y sucesivas formas de legibilidad ${ }^{5}$. De tal modo, la indiscutible preferencia de los(as) encuestados(as) por el "libro", no apunta a un objeto particular, sino que da cuenta, más bien, de la mutación del orden de los discursos, en tanto libros, diarios y revistas se reúnen todos en un mismo soporte: la pantalla, que

4. Esta preferencia se condice con el estudio del CERLALC (2012), "Comportamiento lector y hábitos de lectura”, que señala que el país de América Latina con el menor porcentaje de no lectores de libros es, según sus encuestas, Chile, que en 2011 tenía un 20\% de población no lectora de libros, en contraposición a México, donde se alcanza un 73\% (pág. 7).

5. Roger Chartier atiende a esta doble naturaleza del libro (su "cuerpo y alma"), esto es, su condición tanto de objeto material como de obra intelectual. Ver "La muerte del libro. Orden del discurso y orden de los libros", en Co-herencia, vol. 4, N7, julio-diciembre 2007, págs. 119-129. 
crea una continuidad en la que, por vez primera, ya no se diferencian los discursos a partir de su materialidad.

Respecto a otros formatos, “¿Dónde lees tú?" revela que son los altos ejecutivos y estudiantes los que más leen cómics, mientras las generaciones mayores y aquellos que tienen trabajos ocasionales, prefieren leer revistas. Por su parte, la lectura de diarios aumenta en proporción a la edad, siendo especialmente alta en las generaciones de adultos y adultos mayores.

Según género, cabe destacar que los porcentajes de lectura de diarios y de libros son similares entre hombres y mujeres; no obstante, los primeros leen más cómics y las segundas, más revistas. Es interesante esta distinción en tanto los cómics constituyen un formato de entretención de ficción, desconectado de la realidad, mientras que las revistas, por lo general, traen noticias e información sobre temas relativos a la vida doméstica (hogar, crianza, cocina, uso de la energía, avisos clasificados, etc.), sobre el mercado o la publicidad, y tienen en su público objetivo a las mujeres, agente fundamental en el consumo familiar.

Ahora bien, respecto a los lugares de lectura, se constata la importancia de la lectura íntima o en el hogar, frente a otros espacios como la biblioteca pública. La lectura en la ciudad, específicamente en el transporte público, aparece como un espacio que se abre al desarrollo lector, especialmente en las comunas más habitadas, que tienen mayor distancia o sufren de mayor congestión vial. Así, plazas y parques se presentan como verdaderos oasis en la relación de espacios públicos y lectura, porque en ellos es donde se dan las condiciones que más facilitan el desarrollo de actividades de ocio como la lectura no-obligatoria y, por ende, son un espacio público clave para la integración cultural.

Complementariamente, es probable que, junto a esta mayor individualización en los consumos culturales, específicamente en la lectura, existan fenómenos como la pérdida de atención de los lectores respecto a lugares emblemáticos como las bibliotecas públicas, símbolos de la alfabetización y de una educación "enciclopédica".

A este respecto, es relevante plantear la emergencia de nuevos espacios para la lectura, como el bus y los vagones de Metro, donde los tiempos detenidos o desacelerados se pueden activar por medio de la lectura. En una sociedad con mayor cantidad de personas integradas al mundo laboral y a la ciudad, los medios de transporte cobran una importancia cotidiana mayor. Paralelamente, el aumento en el envejecimiento de la población supone que una cantidad cada vez más importante de personas tendrá tiempo libre o de "esperas" (al igual que el grupo de personas con empleos flexibles u ocasionales). Es importante potenciar la lectura como un mecanismo de vínculo e integración social y generacional.

Respecto a la relación entre lectura y género, la encuesta revela ciertas lógicas invisibles a la actual cartografía de lectura, básicamente el importante rol que poseen las dinámicas familiares, definidas por la distribución sexual del trabajo (ya sea remunerado o de cuidado al interior del hogar), en tanto mediadoras del cómo, cuándo, dónde y qué leen hombres y mujeres, vale decir, en las lógicas de apropiación y usos del libro.

La Encuesta en el Uso del Tiempo del INE (2009) ha constatado amplias brechas entre hombres y mujeres en el uso del tiempo destinado a trabajo remunerado/no 
remunerado, tareas del hogar, cuidado de personas en el hogar, donde la participación femenina es altamente mayor, en comparación con la masculina. En el caso del tiempo dedicado a actividades de ocio y recreación y al uso de las nuevas tecnologías de la información y comunicación, también existen brechas, aunque más leves, a favor de los hombres, quienes tanto durante la semana y sobre todo el fin de semana —que es, habitualmente el tiempo "familiar" - destinarían mayor tiempo a actividades de ocio, recreación o relacionadas con los medios de comunicación (que incluye, en esta encuesta, lectura, ver TV e Internet, entre otras).

De este modo, cabe interrogarse por las barreras en la equidad en el uso del tiempo destinado al ocio y a las actividades culturales o de desarrollo creativo y, en relación a esto, la importancia de iniciativas que permitan mayores equilibrios en los accesos a estos espacios, como serían políticas de afirmación positiva para que las mujeres accedan a mayores tiempos de ocio personal ligados a la cultura.

\section{BIBLIOGRAFÍA}

Chartier, R., "Del códice a la pantalla, trayectorias de lo escrito", en Revista Quimera, N¹50, septiembre de 1996, pág. 49.

_ _La muerte del libro. Orden del discurso y orden de los libros", en Co-herencia, vol. 4, №7, julio-diciembre 2007, págs. 119-129.

Centro Regional para el Fomento del Libro en América Latina y el Caribe (CERLALC). Comportamiento lector y hábitos de lectura. Bogotá, 2012. Disponible en: http://cerlalc.org/

Consejo Nacional de la Cultura y las Artes, Centro de Microdatos, Estudio sobre el comportamiento lector a nivel nacional, Santiago, 2011. Disponible en www.cnca.cl

Segunda Encuesta de Consumo Cultural, Santiago, 2009. Disponible en http://www.cultura. gob.cl/wp-content/uploads/2012/03/Segunda-Encuesta-Nacional-de-Participaci\%C3\%B3ny-Consumo-Cultural.pdf

Darnton, R., "Historia de la lectura", en Formas de hacer historia, Burke, P. (ed.), Madrid: Alianza Editorial, 1996, págs. 177-208.

INE, Boletín Enfoque Estadístico ¿Cómo distribuyen el tiempo hombres y mujeres? Encuesta exploratoria de uso del tiempo en el Gran Santiago, Santiago, 2009. Disponible en http://www.ine.cl/ canales/chile_estadistico/estadisticas_sociales_culturales/encuesta_tiempo_libre/pdf/enfoque_eut_pag.pdf

Manguel, A., "Historia de la lectura", Una historia de la lectura, Madrid: Alianza Editorial, 2009.

Montes, G., La Gran Ocasión: La escuela como sociedad de lectura, Argentina, Plan Nacional de Lectura, Ministerio de Educación, Ciencia y Tecnología, 2007. Disponible en http://planlectura. educ.ar/pdf/La_gran_ocasion.pdf

Observatorio del Libro y la Lectura, Informe Encuesta ¿Dónde lees tú?, Santiago, 2012 y 2013. Disponible en http://www.uchile.cl/portal/extension-y-cultura/vicerrectoria-de-extension/ observatorio-del-libro-y-la-lectura/estudios/84318/habitos-y-practicas-lectoras

Sunkel, G., "Una mirada otra. La cultura desde el consumo", en Mato, D. (comp.), Estudios y otras prácticas intelectuales latinoamericanas en cultura y poder, Caracas, CLACSO, 2002. Disponible en http://bibliotecavirtual.clacso.org.ar/ar/libros/cultura/sunkel.doc 